\title{
GEOMETRY AND REPRESENTATION OF THE SINGULAR SYMPLECTIC FORMS
}

\author{
WOJCIECH DOMITRZ, STANISEAW JANECZKO* \\ and ZBIGNIEW PASTERNAK-WINIARSKI \\ Faculty of Mathematics and Information Science, Warsaw University of Technology \\ Pl. Politechniki 1, 00-661 Warsaw, Poland \\ E-mail:domitrz@mini.pw.edu.pl,janeczko@ise.pw.edu.pl, pastwin@mini.pw.edu.pl
}

\begin{abstract}
In this paper we show to what extent the closed, singular 2-forms are represented, up to the smooth equivalence, by their restrictions to the corresponding singularity set. In the normalization procedure of the singularity set we find the sufficient conditions for the given closed 2 -form to be a pullback of the classical Darboux form. We also find the classification list of simple singularities of the maximal isotropic submanifold-germs in the codimension one Martinet's singular symplectic structures. An example of the exotic singular symplectic structure-germ with no existence of Lagrangian germs is constructed and the singularity theory framework for the pulled back singular symplectic forms is provided.
\end{abstract}

1. Introduction. Let $\omega$ be a closed 2 -form on $R^{2 n}, \omega$ is called a singular form if $\omega^{n}=f \Omega$, where $\Omega$ is the volume form and $f$ is a smooth function $f: R^{2 n} \rightarrow R$ such that $\Sigma_{2}=f^{-1}(0) \neq \emptyset$. In [13], [16] and [8] it was shown that there are five generic types of singularities for germs of closed 2-forms on $R^{4}$. All these generic types, among which one is unstable and has moduli parameters, are determined by the geometry of the pullback $i^{*} \omega$ to the hypersurface of singularities $i: \Sigma_{2} \rightarrow R^{4}$. The positions of $\Sigma_{2}$ and the internal geometry of the form distinguish the singularity types and reconstruct the singular symplectic form itself. There is a straightforward observation that the considered types of forms are represented by the classical Darboux form $\omega_{D}$ on $R^{4}$, namely $\omega=$ $\phi^{*} \omega_{D}$, where $\phi$ is a smooth map-germ. Thus one can formulate immediately the natural questions:

2000 Mathematics Subject Classification: Primary 57R45; Secondary 58A10.

* Present address: Institute of Mathematics, Polish Academy of Sciences, ul. Śniadeckich 8, P.O.Box 21, 00-956 Warsaw, Poland.

Research of the authors supported by KBN grants 3P03A06910, 2P03A02017 and partially supported by EC IHP programme Contract number HPRN-CT-00271.

The paper is in final form and no version of it will be published elsewhere. 
1. How do the invariants of the geometry of the singularity surface $\Sigma_{2}$ distinguish and describe the equivalence classes of the closed 2-forms?

2. Can any closed 2-form be represented as a pullback from the canonical Darboux form?

3. Is the geometry of isotropic varieties for the singular symplectic forms determined by the isotropic varieties in the Darboux case?

In Section 2 we show to what extent the closed 2-form is represented by its restriction to the singularity hypersurface, moreover we express the closed 2 -forms on $R^{2 n}$, with the singularity set being a smooth hypersurface, by the specific forms on the singularity set. The similar problems for singular contact structures are considered in [9], [10].

An answer for the second question is obtained in Section 3. At first it is shown that all diagonal closed 2-forms are represented by the canonical pullback, then the sufficient conditions are found for the general singular closed 2-form to be a pullback from the Darboux form. In this section we also show that for the important class of closed 2-forms their canonical representation exists.

Then in Section 4 we show the possible pathological difference between singular symplectic structures and symplectic structures. For that we construct the singular exotic symplectic form, which cannot be reduced to the diagonal one and has no maximal isotropic subspace passing through the chosen source point of the germ. This suggests the nontriviality of the classification problem for the maximal isotropic germs of surfaces in the singular symplectic structures (as is also pointed out in $[11,12]$ ). Thus as an introductory approach, the list of simple normal forms of maximal isotropic germs in the simplest $\Sigma_{20}$ Martinet's singular symplectic structure is obtained.

2. Singularity sets for the degenerate symplectic structures. Let $M$ be an $m$-dimensional smooth manifold $(m \geq 2)$ and let $\omega$ be an arbitrary 2 -form on $M$. The integer $\left.\operatorname{rank} \omega\right|_{x}=2 s(x)$ defined by

$$
\left(\left.\omega\right|_{x}\right)^{s(x)} \neq 0, \quad\left(\left.\omega\right|_{x}\right)^{s(x)+1}=0,
$$

is called the rank of $\omega$ at $x \in M$. For any $x \in M$ there exists a basis $\mathcal{B}=\left(e_{1}, e_{2}, \ldots, e_{m}\right)$ of the space $T_{x} M$ such that

$$
\left.\omega\right|_{x}=\sum_{k=1}^{s(x)} e_{2 k-1}^{*} \wedge e_{2 k}^{*},
$$

where $\left(e_{1}^{*}, \ldots e_{m}^{*}\right)$ is the basis of $T_{x}^{*} M$ dual to $\mathcal{B}$ (see [4], I, Theorem 5.1). Any such $\mathcal{B}$ will be called a canonical basis for the form $\omega$ at the point $x$. If $x$ is fixed then there exist a neighborhood $U$ of $x$ in $M$ and coordinates $x_{1}, \ldots, x_{m}$ on $U$ such that

$$
e_{1}=\left.\frac{\partial}{\partial x_{1}}\right|_{x}, e_{2}=\left.\frac{\partial}{\partial x_{2}}\right|_{x}, \ldots, e_{m}=\left.\frac{\partial}{\partial x_{m}}\right|_{x} .
$$

Then, in this basis, we have

$$
\left.\omega\right|_{x}=\sum_{k=1}^{s(x)} d x_{2 k-1} \wedge d x_{2 k} .
$$


We will call $x_{1}, x_{2}, \ldots, x_{m}$ the canonical coordinates for $\omega$ at $x \in M$.

If $M$ is an even-dimensional manifold then there are canonical coordinates at $x \in M$, $(p, q)=\left(p_{1}, \ldots, p_{n}, q_{1}, \ldots, q_{n}\right)$, such that

$$
\left.\omega\right|_{x}=\sum_{k=1}^{s(x)} d p_{k} \wedge d q_{k} .
$$

Now we introduce the following notion

Definition 1. The set

$$
\Sigma_{2}(\omega)=\left\{x \in M:\left.\operatorname{rank} \omega\right|_{x}<\operatorname{dim} M\right\}
$$

is called the singularity set of $\omega$.

Let $\omega$ be a closed 2 -form on $R^{2 n}$. Let $(p, q)=\left(p_{1}, \ldots, p_{n}, q_{1}, \ldots, q_{n}\right)$ be a coordinate system on $R^{2 n}$. $\Omega=d p_{1} \wedge d q_{1} \wedge \ldots \wedge d p_{n} \wedge d q_{n}$ is the volume form. Then $\omega^{n}=f \Omega$, where $f$ is a smooth function on $R^{2 n}$. The singularity set of $\omega$ is the zero level set of $f$.

Proposition 1. Let $S$ be a closed subset of $R^{2 n}$. Then there exists a closed 2-form $\omega$ such that $S=\Sigma_{2}(\omega)$.

Proof. There exists a smooth function $f$ on $R^{2 n}$ such that $S=f^{-1}(0)$. We consider the 1 -form

$$
\alpha=\sum_{i=2}^{n} p_{i} d q_{i}+\left(\int_{0}^{p_{1}} f\left(t, p_{2}, \ldots, p_{n}, q_{1}, \ldots, q_{n}\right) d t\right) d q_{1} .
$$

Let $\omega=d \alpha$. Then it is easy to check that $\Sigma_{2}(\omega)=f^{-1}(0)=S$.

We assume that zero is a regular value of $f$ in the formula $\omega^{n}=f \Omega$. Hence $\Sigma_{2}(\omega)=$ $f^{-1}(0)$ is a smooth hypersurface of codimension 1 in $R^{2 n}$. We may find a coordinate system $(p, q)$ on $R^{2 n}$ such that locally $f(p, q)=p_{1}$ (cf. [13]).

It is obvious that if two singular closed 2 -forms are equivalent then their pullbacks to their singularity sets are equivalent. We want to consider the inverse problem. Now we have the following straightforward lemma.

LEMMA 1. Let $\omega_{0}$ and $\omega_{1}$ be two germs of closed 2 -forms on $R^{2 n}$ at 0 , such that $\omega_{0}^{n} / \Omega$ and $\omega_{1}^{n} / \Omega$ are regular functions at 0 . Assume that $H$ is a common singularity hypersurface of both these forms. Then

$$
\omega_{0}^{n}=f \Omega, \quad \omega_{1}^{n}=(a+g) f \Omega,
$$

where $a \neq 0,\left.d f\right|_{0} \neq 0$ and $f, g$ are smooth function-germs vanishing at 0 .

Firstly we assume that these singular 2-forms are equal on their singularity set. Then we obtain the following result.

TheOREM 1. Let $\omega_{0}$ and $\omega_{1}$ be two germs at 0 of closed 2-forms on $R^{2 n}$ with the common singularity hypersurface $H=\{f=0\}, \omega_{0}^{n}=f \Omega, \omega_{1}^{n}=f(a+\tilde{g}) \Omega,\left.d f\right|_{0} \neq 0$, where $\tilde{g}$ is a smooth function-germ, $\tilde{g}(0)=0, a>0,\left.\omega_{0}\right|_{T_{H} R^{2 n}}=\left.\omega_{1}\right|_{T_{H} R^{2 n}}=\tilde{\omega}$.

Then there exists a diffeomorphism-germ $\Phi:\left(R^{2 n}, 0\right) \rightarrow\left(R^{2 n}, 0\right)$ such that

$$
\Phi^{\star} \omega_{1}=\omega_{0}
$$

and $\left.\Phi\right|_{H}=\operatorname{Id}_{H}$. 
Proof. First we simplify the forms $\omega_{0}$ and $\omega_{1}$. We find the local coordinate system such that $\omega_{0}^{n}=p_{1} \Omega, \omega_{1}^{n}=p_{1}(a+g) \Omega$, where $g$ is a smooth function-germ, $g(0)=0$. By assumptions, we have $\omega_{i}=p_{1} \alpha_{i}+\tilde{\omega}$, where $\tilde{\omega}=\left.\omega_{i}\right|_{T_{\left\{p_{1}=0\right\}} R^{2 n}}$ for $i=0,1$. Then further on we use the Moser homotopy method (see [15]). Let $\omega_{t}=t \omega_{1}+(1-t) \omega_{0}$, for $t \in[0,1]$.

We assume that $\Phi_{t}^{\star} \omega_{t}=\omega_{0}$, for $t \in[0,1], \Phi_{0}=\mathrm{Id}$. Differentiating the above homotopy equation by $t$, we obtain

$$
\left.d\left(V_{t}\right\rfloor \omega_{t}\right)=\omega_{0}-\omega_{1}=p_{1}\left(\alpha_{0}-\alpha_{1}\right),
$$

where $V_{t}=\frac{d}{d t} \Phi_{t}$. Now we prove the following

LEMMA 2. If $p_{1} \alpha$ is a closed 2-form on $R^{2 n}$ then there exists a 1-form $\beta$ such that $p_{1} \alpha=d\left(p_{1}^{2} \beta\right)$.

Proof of Lemma 2. One can show that there exists a 1-form $\gamma$ such that $p_{1} \alpha=$ $d\left(p_{1} \gamma\right)=d p_{1} \wedge \gamma+p_{1} d \gamma$ (see [2]). Therefore $\left.d p_{1} \wedge \gamma\right|_{T_{\left\{p_{1}=0\right\}} R^{2 n}}=0$. Hence there exist a 1 -form $\delta$ and a smooth function $f$ such that $\gamma=p_{1} \delta+f d p_{1}$. If we take $\beta=\delta-\frac{d f}{2}$ then

$$
p_{1} \alpha=d\left(p_{1} \gamma-d\left(\frac{p_{1}^{2} f}{2}\right)\right)=d\left(p_{1}^{2} \beta\right)
$$

which finishes the proof of Lemma 2.

Let us notice that $p_{1}\left(\alpha_{0}-\alpha_{1}\right)=\omega_{1}-\omega_{0}$ is closed. By the above lemma we have

$$
\left.V_{t}\right\rfloor \omega_{t}=p_{1}^{2} \beta
$$

Now we compute

$$
\omega_{i}^{n}=\left(p_{1} \alpha_{i}+\tilde{\omega}\right)^{n}=\tilde{\omega}^{n}+p_{1} \sum_{k=1}^{n}\left(\begin{array}{l}
n \\
k
\end{array}\right) p_{1}^{k-1} \alpha_{i}^{k} \wedge \tilde{\omega}^{n-k} .
$$

But $\left.\omega_{i}^{n}\right|_{T_{\left\{p_{1}=0\right\}} R^{2 n}}=0$. This clearly forces $\tilde{\omega}^{n}=0$. By the above formula we get

$$
n \alpha_{0} \wedge \tilde{\omega}^{n-1}=\Omega-p_{1} \sum_{k=2}^{n}\left(\begin{array}{l}
n \\
k
\end{array}\right) p_{1}^{k-2} \alpha_{0}^{k} \wedge \tilde{\omega}^{n-k}
$$

and

$$
n \alpha_{1} \wedge \tilde{\omega}^{n-1}=(a+g) \Omega-p_{1} \sum_{k=2}^{n}\left(\begin{array}{l}
n \\
k
\end{array}\right) p_{1}^{k-2} \alpha_{1}^{k} \wedge \tilde{\omega}^{n-k} .
$$

These equations imply the formula

$$
\begin{aligned}
\omega_{t}^{n}= & \left(p_{1}\left(t \alpha_{1}+(1-t) \alpha_{0}\right)+\tilde{\omega}\right)^{n} \\
= & p_{1}\left(t n \alpha_{1} \wedge \tilde{\omega}^{n-1}+(1-t) n \alpha_{0} \wedge \tilde{\omega}^{n-1}\right)+\sum_{k=2}^{n}\left(\begin{array}{l}
n \\
k
\end{array}\right) p_{1}^{k}\left(t \alpha_{1}+(1-t) \alpha_{0}\right)^{k} \wedge \tilde{\omega}^{n-k} \\
= & p_{1}(1+t(a+g-1)) \Omega \\
& \quad+p_{1}^{2} \sum_{k=2}^{n}\left(\begin{array}{l}
n \\
k
\end{array}\right) p_{1}^{k-2}\left(\left(t \alpha_{1}+(1-t) \alpha_{0}\right)^{k}-t \alpha_{1}^{k}-(1-t) \alpha_{0}^{k}\right) \wedge \tilde{\omega}^{n-k} .
\end{aligned}
$$

From (7) we obtain

$$
\omega_{t}^{n}=p_{1}\left(1+t(a+g-1)+p_{1} h_{t}\right) \Omega
$$


where $h_{t}$ is a smooth function. Let us notice that $(1+t(a+g(0)-1)) \neq 0$ for $a>0$ and for $t \in[0,1]$. Therefore $\omega_{t}$ has the same singularity type for every $t \in[0,1]$. The set of singular points of $\omega_{t}$ is nowhere dense, therefore by direct algebraic calculation, it is easy to see that equation (6) is equivalent to the equation

$$
\left.V_{t}\right\rfloor \omega_{t}^{n}=n p_{1}^{2} \beta \wedge \omega_{t}^{n-1} .
$$

Combining this equation with equation (8) we obtain

$$
\left.V_{t}\right\rfloor\left(1+t(a+g-1)+p_{1} h_{t}\right) \Omega=n p_{1} \beta \wedge \omega_{t}^{n-1} .
$$

We can find a smooth vector field $V_{t}$ that satisfies (10) and $\left.V_{t}\right|_{H}=0$, because $(1+t(a+g(0)-1)) \neq 0$ and the right hand side of (10) vanishes on $H$. Hence there exists a diffeomorphism $\Phi_{t}$ such that $\Phi_{t}^{\star} \omega_{t}=\omega_{0}$ for $t \in[0,1]$ and $\left.\Phi_{t}\right|_{H}=\operatorname{Id}_{H}$. This completes the proof of Theorem 1 .

Now we define

$$
\iota:\left\{p_{1}=0\right\} \ni\left(p_{2}, \ldots, p_{n}, q_{1}, \ldots, q_{n}\right) \mapsto\left(0, p_{2}, \ldots, p_{n}, q_{1}, \ldots, q_{n}\right) \in R^{2 n}
$$

and

$$
\pi: R^{2 n} \ni\left(p_{1}, p_{2}, \ldots, p_{n}, q_{1}, \ldots, q_{n}\right) \mapsto\left(p_{2}, \ldots, p_{n}, q_{1}, \ldots, q_{n}\right) \in\left\{p_{1}=0\right\} .
$$

In general, using the above theorem, we reduce $\omega$ to the following prenormal form.

Proposition 2. If $\omega^{n}=f \Omega, f(0)=0$ and $\left.d f\right|_{0} \neq 0$, then there exists a germ of a diffeomorphism $\Phi:\left(R^{2 n}, 0\right) \rightarrow\left(R^{2 n}, 0\right)$ such that

$$
\Phi^{\star} \omega=d\left(p_{1} \pi^{\star} \alpha+p_{1}^{2} \pi^{\star} \beta\right)+\pi^{\star} \sigma,
$$

where $\sigma=\iota^{\star} \omega$ and $\alpha, \beta$ are germs of 1 -forms on $\left\{p_{1}=0\right\}$ satisfying the conditions

1. $\alpha \wedge(\sigma)^{n-1}=0$,

2. $\left.(2 \beta \wedge \sigma+(n-1) \alpha \wedge d \alpha) \wedge(\sigma)^{n-2}\right|_{0} \neq 0$.

Proof. It is easy to see that there exists 1 -form $\gamma$ such that $\omega=d\left(p_{1} \gamma\right)+\pi^{\star} \sigma$. It is clear that we can write $\gamma$ in the form $\gamma=\pi^{\star} \alpha+p_{1} \delta+g d p_{1}$, where $\alpha$ is a germ of a 1-form on $\left\{p_{1}=0\right\}, g$ is a function-germ and $\delta$ is a germ of a 1-form. Then

$$
d\left(p_{1}\left(p_{1} \delta+g d p_{1}\right)\right)=p_{1}\left(2 d p_{1} \wedge \delta+p_{1} d \delta+d g \wedge d p_{1}\right) .
$$

By Lemma 2 we have $\omega=d\left(p_{1} \pi^{\star} \alpha\right)+d\left(p_{1}^{2} \theta\right)+\pi^{\star} \sigma$. Now we write $\theta$ in the form $\theta=$ $\pi^{\star} \beta+p_{1} \eta+h d p_{1}$, where $\beta$ is a germ of a 1 -form on $\left\{p_{1}=0\right\}, h$ is a function-germ and $\eta$ is a germ of a 1 -form. Then

$$
\omega=d\left(p_{1} \pi^{\star} \alpha+p_{1}^{2} \pi^{\star} \beta\right)+\pi^{\star} \sigma+p_{1}^{2}\left(3 d p_{1} \wedge \eta+p_{1} d \eta+d h \wedge d p_{1}\right) .
$$

Let $\omega_{0}=d\left(p_{1} \pi^{\star} \alpha+p_{1}^{2} \pi^{\star} \beta\right)+\pi^{\star} \sigma$.

It is easy to see that

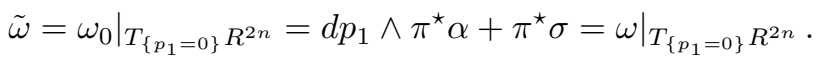

Now we calculate

$$
\omega_{0}^{n}=\left(\omega+p_{1}^{2} \zeta\right)^{n}=p_{1} \Omega+p_{1}^{2} \sum_{k=1}^{n}\left(\begin{array}{l}
n \\
k
\end{array}\right) p_{1}^{2(k-1)} \zeta^{k} \wedge \omega^{n-k}=p_{1}\left(1+p_{1} r\right) \Omega
$$


where $\zeta=-\left(3 d p_{1} \wedge \eta+p_{1} d \eta+d h \wedge d p_{1}\right)$ and $r$ is a smooth function-germ. Therefore by Theorem 1 we have $\Phi^{\star} \omega=\omega_{0}$. Then we calculate again

$$
\begin{aligned}
\omega_{0}^{n}=n d p_{1} \wedge & \pi^{\star} \alpha \wedge\left(\pi^{\star} \sigma\right)^{n-1} \\
& +n p_{1} d p_{1} \wedge\left(2 \pi^{\star} \beta \wedge \pi^{\star} \sigma+(n-1) \pi^{\star} \alpha \wedge d \pi^{\star} \alpha\right) \wedge\left(\pi^{\star} \sigma\right)^{n-2}+p_{1}^{2} v \Omega,
\end{aligned}
$$

where $v$ is a smooth function-germ. Combining the above formula with (11) we obtain conditions 1 and 2 of Proposition 2.

Now we show the lower bound for the rank of the pullback of the generic singular closed 2-form onto its singularity set.

Corollary 1. If $\omega^{n}=f \Omega, f(x)=0,\left.d f\right|_{x} \neq 0$ and $\iota^{\star} \omega=\sigma$, where $\iota:\{f=0\} \hookrightarrow$ $R^{2 n}$, then $\left.\operatorname{rank} \sigma\right|_{x}=2 n-2$ or $\left.\operatorname{rank} \sigma\right|_{x}=2 n-4$.

Proof. We may assume that $\omega^{n}=p_{1} \Omega$ and $x=0$. By Proposition 2 we have $\left.(2 \beta \wedge \sigma+(n-1) \alpha \wedge d \alpha) \wedge(\sigma)^{n-2}\right|_{0} \neq 0$. This implies $\left.\sigma^{n-2}\right|_{0} \neq 0$, where $\sigma=\iota^{\star} \omega$. Therefore $2 n-4 \leq\left.\operatorname{rank} \sigma\right|_{0}<2 n$.

REMARK 1. Let $\omega$ satisfy the assumptions of Corollary 1 at $x=0$. The condition rank $\left.\sigma\right|_{0}=2 n-2$ is equivalent to the transversality of

$$
\left.\operatorname{ker} \omega=\left\{v \in T_{f^{-1}(0)} R^{2 n}: v\right\rfloor \omega=0\right\}
$$

to $\Sigma_{2}(\omega)=f^{-1}(0)$ at 0 . If $\omega$ satisfies the above condition then there exists a germ of a diffeomorphism $\Phi:\left(R^{2 n}, 0\right) \rightarrow\left(R^{2 n}, 0\right)$ such that

$$
\Phi^{\star} \omega=p_{1} d q_{1} \wedge d q_{1}+\sum_{i=2}^{n} d p_{i} \wedge d q_{i},
$$

see [13]. This singular closed 2-form is called $\Sigma_{20}$ Martinet's singular symplectic form. Thus the equivalence class of $\omega$ is determined by its pullback to the singularity hypersurface if $\left.\operatorname{rank} \iota^{\star} \omega\right|_{0}=2 n-2$. Therefore the most interesting case is $\left.\operatorname{rank} \iota^{\star} \omega\right|_{0}=2 n-4$.

Now we show that the closed 2-form with regular singularity hypersurface is determined by the pullback to the hypersurface and by a 1-form on that hypersurface.

Theorem 2. Let $\omega^{n}=f \Omega, f(0)=0$ and $\left.d f\right|_{0} \neq 0$. If rank $\left.\iota^{\star} \omega\right|_{0}=2 n-4$ then there exists a germ of a diffeomorphism $\Phi:\left(R^{2 n}, 0\right) \rightarrow\left(R^{2 n}, 0\right)$ such that

$$
\Phi^{\star} \omega=d\left(p_{1} \pi^{\star} \alpha\right)+\pi^{\star} \sigma
$$

where $\sigma=\iota^{\star} \omega$ and $\alpha$ is a germ of 1 -form on $\left\{p_{1}=0\right\}$ such that

1. $\alpha \wedge \sigma^{n-1}=0$,

2. $\left.\alpha \wedge d \alpha \wedge \sigma^{n-2}\right|_{0} \neq 0$.

Proof. By Proposition 2, $\omega=d\left(p_{1} \pi^{\star} \alpha\right)+d\left(p_{1}^{2} \pi^{\star} \beta\right)+\pi^{\star} \sigma$. Then

$$
\omega^{n}=2 n p_{1} d p_{1} \wedge \pi^{\star} \beta \wedge \pi^{\star}\left(\sigma^{n-1}\right)+n(n-1) p_{1} d p_{1} \wedge \pi^{\star} \alpha \wedge d \pi^{\star} \alpha \wedge \pi^{\star}\left(\sigma^{n-2}\right)+p_{1}^{2} v \Omega,
$$

where $v$ is a smooth function-germ at 0 . From $\left.\sigma^{n-1}\right|_{0}=0$ we have

$$
\omega^{n}=n(n-1) p_{1} d p_{1} \wedge \pi^{\star} \alpha \wedge d \pi^{\star} \alpha \wedge \pi^{\star}\left(\sigma^{n-2}\right)+p_{1} g \Omega,
$$


where $g$ is a smooth function-germ at 0 such that $g(0)=0$. Therefore $\left.\alpha \wedge d \alpha \wedge \sigma^{n-2}\right|_{0} \neq 0$. Let

$$
\omega_{0}=d\left(p_{1} \pi^{\star} \alpha\right)+\pi^{\star} \sigma
$$

Then

$$
\omega_{0}^{n}=n(n-1) p_{1} d p_{1} \wedge \pi^{\star} \alpha \wedge d \pi^{\star} \alpha \wedge \pi^{\star}\left(\sigma^{n-2}\right)+p_{1} h \Omega,
$$

where $h$ is a smooth function-germ at 0 such that $h(0)=0$. One can check that

$$
\tilde{\omega}=\left.\omega_{0}\right|_{T_{\left\{p_{1}=0\right\}} R^{2 n}}=d p_{1} \wedge \pi^{\star} \alpha+\pi^{\star} \sigma=\left.\omega\right|_{T_{\left\{p_{1}=0\right\}} R^{2 n}} .
$$

Therefore by Theorem 1 there exists a germ of a diffeomorphism $\Theta:\left(R^{2 n}, 0\right) \rightarrow\left(R^{2 n}, 0\right)$ such that $\Theta^{\star} \omega=\omega_{0}$ and $\left.\Theta\right|_{\left\{p_{1}=0\right\}}=\operatorname{Id}_{\left\{p_{1}=0\right\}}$.

Let us assume that $n=2$. $\omega$ is a germ of a smooth closed 2 -form on $R^{4}$ at 0 . From Theorem 2 we obtain

Proposition 3. Let $\omega^{2}=f \Omega, f(0)=0$ and $\left.d f\right|_{0} \neq 0$. If $\left.\operatorname{rank} \iota^{\star} \omega\right|_{0}=0$ then there exists a germ of a diffeomorphism $\Phi:\left(R^{4}, 0\right) \rightarrow\left(R^{4}, 0\right)$ such that

$$
\Phi^{\star} \omega=d\left(p_{1} \pi^{\star} \alpha\right)+\pi^{\star} \sigma
$$

where $\sigma=\iota^{\star} \Phi^{\star} \omega$ is a germ of a closed 2 -form on $\left\{p_{1}=0\right\}$ such that $\alpha \wedge \sigma=0$ and in this four-dimensional case $\alpha$ is a contact form on $\left\{p_{1}=0\right\}$.

REMARK 2. The set

$$
\Sigma_{22}(\omega)=\left\{x \in \Sigma_{2}(\omega):\left.\operatorname{rank} \omega\right|_{x}=0\right\},
$$

consists of the points where ker $\omega$ is tangent to $\Sigma_{2}(\omega)$. Generically $\Sigma_{22}(\omega)$ has codimension 2 in $\Sigma_{2}(\omega)$. If ker $\omega$ is transversal to $\Sigma_{22}(\omega)$ at 0 in $\Sigma_{2}(\omega)$ then there exists a germ of a diffeomorphism $\Phi:\left(R^{4}, 0\right) \rightarrow\left(R^{4}, 0\right)$ such that

$$
\Phi^{\star} \omega=d\left(p_{1}-p_{2}^{2} / 2\right) \wedge d q_{1}+d\left(p_{1} p_{2} \pm q_{1} q_{2}-p_{2}^{3} / 3\right) \wedge d q_{2},
$$

see [16]. These singular closed 2-forms are called elliptic and hyperbolic $\Sigma_{220}$ Martinet's singular symplectic forms. The symplectic form, the $\Sigma_{20}$-type form, and both types of $\Sigma_{220}$ forms are locally stable on $R^{4}$. Golubitsky and Tischler [8] have shown that there are no more locally stable closed 2 -forms on $R^{4}$. They used the contact form $\alpha$ (see Proposition 3) on a singularity hypersurface to prove this result.

By Proposition 3, it is easy to see that all germs of closed 2-forms on $R^{4}$ that vanish on their singularity hypersurface are equivalent.

Corollary 2. Let $\omega^{2}=f \Omega, f(0)=0$ and $\left.d f\right|_{0} \neq 0$. If $\iota^{\star} \omega=0$ then there exists a germ of a diffeomorphism $\Phi:\left(R^{4}, 0\right) \rightarrow\left(R^{4}, 0\right)$ such that

$$
\Phi^{\star} \omega=d\left(p_{1}\left(d q_{1}+p_{2} d q_{2}\right)\right) .
$$

3. Canonical representation of a closed 2-form. Let $(p, q)=\left(p_{1}, \ldots, p_{n}, q_{1}, \ldots\right.$, $\left.\ldots, q_{n}\right)$ be a coordinate system on $R^{2 n}$. We denote by $\omega_{D}$ the Darboux 2 -form, i.e.

$$
\omega_{D}:=\sum_{j=1}^{n} d p_{j} \wedge d q_{j} .
$$

The celebrated Darboux theorem states (see [17], [13], [2]): 
TheOREM 3. Let $\omega$ be a germ at $x$ of a closed and nondegenerate 2-form on a $2 n$-dimensional manifold $M$. Then there exists a germ of a diffeomorphism $\Phi:(M, x) \rightarrow$ $\left(R^{2 n}, 0\right)$ such that

$$
\omega=\Phi^{\star} \omega_{D} .
$$

In this section we want to generalize this theorem to the case when $x \in \Sigma_{2}(\omega)$. We are looking for necessary and sufficient conditions for $\omega$ to be of the form (13), where $\Phi:(M, x) \rightarrow\left(R^{2 n}, 0\right)$ is a smooth map (which is singular at $x$ ). Note that if $f$ is an arbitrary $C^{\infty}$-function on $R^{2 n}$ and $\omega=d \alpha$ where the 1 -form $\alpha$ is given by (5) and $\Sigma_{2}(\omega)=f^{-1}(0)$, then the suitable map $\Phi$ exists. Namely, we can take

$$
\begin{aligned}
& \Phi\left(p_{1}, \ldots, p_{n}, q_{1}, \ldots, q_{n}\right) \\
& \quad=\left(\int_{0}^{p_{1}} f\left(t, p_{2}, \ldots, p_{n}, q_{1}, \ldots, q_{n}\right) d t, p_{2}, \ldots, p_{n}, q_{1}, \ldots, q_{n}\right) .
\end{aligned}
$$

Now we consider the case when there are coordinates $(p, q)=\left(p_{1}, \ldots, p_{n}, q_{1}, \ldots, q_{n}\right)$ such that $\omega$ can be written in the following way

$$
\omega(p, q)=\sum_{j=1}^{n} \omega_{j}(p, q) d p_{j} \wedge d q_{j} .
$$

We will call closed 2-forms of this type the diagonal forms.

If $\omega$ can be written in the form (15) then for any $j=1,2, \ldots, n$ the function-germ $\omega_{j}$ depends only on $p_{j}$ and $q_{j}$ i.e. $\omega_{j}(p, q)=\omega_{j}\left(p_{j}, q_{j}\right)$. Indeed, since

$$
d \omega=\sum_{k \neq j}\left(\frac{\partial \omega_{j}}{\partial q_{k}} d q_{k} \wedge d p_{j} \wedge d q_{j}+\frac{\partial \omega_{j}}{\partial p_{k}} d p_{k} \wedge d p_{j} \wedge d q_{j}\right)=0
$$

and the elements $d q_{k} \wedge d p_{j} \wedge d q_{j}$ and $d p_{k} \wedge d p_{j} \wedge d q_{j}, j, k=1,2, \ldots, n, j \neq k$, are linearly independent, we deduce that $\frac{\partial \omega_{j}}{\partial q_{k}}=\frac{\partial \omega_{j}}{\partial p_{k}} \equiv 0$ for $j \neq k$.

Let $\omega$ be a germ at $x$ of a closed 2 -form on a $2 n$-dimensional manifold $M$. Suppose that there exists a chart $\Psi=\left(p_{1}, \ldots, p_{n}, q_{1}, \ldots, q_{n}\right): U \rightarrow R^{2 n}$ on $M$, such that the equality (15) holds. If we take $\bar{\Psi}=\Psi-\Psi(x)$ instead of $\Psi$ then we have that $\bar{\Psi}(x)=0$. Let a $2 n$-dimensional cube $(-a ; a)^{2 n}$ be contained in $\bar{\Psi}(U)$ and let $V:=\bar{\Psi}^{-1}\left((-a ; a)^{2 n}\right)$. Define

$$
\begin{gathered}
P_{j}(p, q):=\int_{0}^{p_{j}} \omega_{j}\left(t, q_{j}\right) d t \\
Q_{j}(p, q):=q_{j}, \quad j=1,2, \ldots, n, \quad(p, q) \in(-a ; a)^{2 n}, \\
(P, Q):=\left(P_{1}, \ldots, P_{n}, Q_{1}, \ldots, Q_{n}\right) .
\end{gathered}
$$

Then we have the map-germ $\Phi=\left.(P, Q) \circ \bar{\Psi}\right|_{V}$, such that $\omega=\Phi^{\star} \omega_{D}$.

Let $\omega$ be a diagonal 2 -form for some chart $\Psi$ (see (15)). Then

$$
\omega^{n}(p, q)=\omega_{1}\left(p_{1}, q_{1}\right) \cdot \ldots \cdot \omega_{n}\left(p_{n}, q_{n}\right) \Omega(p, q),
$$

where $\Omega=d p_{1} \wedge d q_{1} \wedge \ldots \wedge d p_{n} \wedge d q_{n}$. If $x \in \Sigma_{2}(\omega)$ then there exists $m \in\{1,2, \ldots, n\}$ such that $\omega_{m}\left(p_{m}(x), q_{m}(x)\right)=0$. Hence $\Sigma_{2}(\omega)$ contains a submanifold $S:=\Psi^{-1}(\{(p, q) \in$ $\left.\left.R^{2 n}: p_{m}=p_{m}(x), q_{m}=q_{m}(x)\right\}\right)$ of codimension two. 
EXAMPLE 1. Let

$$
f\left(p_{1}, p_{2}, q_{1}, q_{2}\right):=p_{1}^{2}+p_{2}^{2}+q_{1}^{2}+q_{2}^{2}, \quad(p, q) \in R^{4},
$$

and let $\omega=d \alpha$, where $\alpha$ is the 1-form defined in (5). Since $\Sigma_{2}(\omega)=\{0\} \subset R^{4}$ does not contain any submanifold of codimension two we obtain that a chart $\Psi=(p, q)$ such that $\omega$ has a diagonal form (15) does not exist. On the other hand there exists a map $\Phi$ such that $\omega$ is the pullback from the Darboux form $\omega_{D}$ (see (14)).

Another example of a closed 2-form $\omega$ which does not fulfil (15) is provided in the next section (see Example 2).

Now we give a sufficient condition for $\omega$ to be of the form of pullback (13). To do this we should recall some results concerning the Pfaffian systems (cf. [4]). Let $\alpha$ be a smooth 1-form on a manifold $M$. The Pfaff problem concerning $\alpha$ is the problem of finding all submanifolds $S$ of $M$ for which

$$
\iota_{S}^{*} \alpha=0,
$$

where $\iota_{S}: S \rightarrow M$ is the natural embedding of $S$ into $M$. The integer $r(x)$ defined by

$$
\left.\left(\left.d \alpha\right|_{x}\right)^{r(x)} \wedge \alpha\right|_{x} \neq 0, \quad\left(\left.d \alpha\right|_{x}\right)^{r(x)+1}=0
$$

is called the rank of the equation (16) at a point $x \in M$ (see [4], p. 38). In this paper we will call it the Pf-rank of the form $\alpha$ at a point $x \in M$. The Pf-rank is invariant under the multiplication $\alpha \rightarrow g \alpha$, where $g \in C^{\infty}(M)$ and $g(x) \neq 0$ for any $x \in M$. We need the following result which is known in the theory of Pfaffian systems.

THEOREM 4. Assume that a germ at $x$ of a 1 -form $\alpha$ on a manifold $M$ has a constant Pf-rank $r$. Then there exist a coordinate system $w_{1}, \ldots, w_{m}(m=\operatorname{dim} M)$ and a smooth function-germ $g$ at $x$ such that $g(x) \neq 0$ and

$$
\alpha=g\left(d w_{1}+w_{2} d w_{3}+\ldots+w_{2 r} d w_{2 r+1}\right) .
$$

The proof of this theorem can be found in [4] (Chapter II, page 38, Theorem 3.1).

Using this theorem we can prove the main result of the section.

TheOREM 5. Let $\omega$ be a germ at $x$ of a closed 2-form on a $2 n$-dimensional manifold $M$. Suppose that there exist:

(i) a smooth function-germ $f$ at $x$ on $M$,

(ii) a germ $\alpha$ at $x$ of a smooth 1 -form on $M$ such that $\omega=d(f \alpha)$ and $\alpha$ has a constant Pf-rank $r$.

Then there exists a smooth map-germ $\Phi:(M, x) \rightarrow\left(R^{2 n}, 0\right)$ such that $\omega=\Phi^{\star} \omega_{D}$.

Proof. Since $(d \alpha)^{r} \wedge \alpha \neq 0$, we deduce that $r<n$. By Theorem 4 , there exist a coordinate system $w_{1}, \ldots, w_{2 n}$ in a neighborhood $V$ of $x$ and a smooth function $g$ on $V$ such that equality (18) holds. Then

$$
\begin{aligned}
\omega=d\left(f g \left(d w_{1}+w_{2} d w_{3}\right.\right. & \left.\left.+\ldots+w_{2 r} d w_{2 r+1}\right)\right) \\
& =d(f g) \wedge d w_{1}+d\left(f g w_{2}\right) \wedge d w_{3}+\ldots+d\left(f g w_{2 r}\right) \wedge d w_{2 r+1}
\end{aligned}
$$

and $\omega=\Phi^{\star} \omega_{D}$ for

$$
\Phi\left(w_{1}, \ldots, w_{2 n}\right):=(\underbrace{f g, f g w_{2}, f g w_{4}, \ldots, f g w_{2 r}, 0, \ldots, 0}_{n}, \underbrace{w_{1}, w_{3}, \ldots, w_{2 r+1}, 0, \ldots, 0}_{n}) .
$$


Corollary 3. If $\left.\omega\right|_{x} ^{n-1} \neq 0$ then there exists $\Phi:(M, x) \rightarrow\left(R^{2 n}, 0\right)$ such that $\omega=\Phi^{\star} \omega_{D}$.

Proof. If $\left.\omega\right|_{x} ^{n} \neq 0$ then the result follows immediately from Theorem 3. Suppose that $\left.\omega\right|_{x} ^{n}=0$, i.e. $\operatorname{rank} \omega_{x}=2(n-1)$. Let $(p, q)$ be the canonical coordinates for $\omega$ at $x$. Then

$$
\left.\omega\right|_{x}=\sum_{k=1}^{n-1} d p_{k} \wedge d q_{k}
$$

(see (4)) which implies

$$
\left.\omega\right|_{x} ^{n-1}=(n-1) ! d p_{1} \wedge d q_{1} \wedge \ldots \wedge d p_{n-1} \wedge d q_{n-1} .
$$

Let $\theta$ be a 1 -form in a neighborhood $U_{0}$ of $x$ such that $d \theta=\omega$. If we put

$$
\alpha:=\theta-\theta_{x}+d p_{n},
$$

where $\theta_{x}$ has constant coefficients on $U_{0}$ (with respect to the coordinates $(p, q)$ ), then $d \alpha=\omega$ and $\alpha_{x}=d p_{n}$. This gives

$$
\left(\omega^{n-1} \wedge \alpha\right)_{x}=(n-1) ! d p_{1} \wedge d q_{1} \wedge \ldots \wedge d p_{n-1} \wedge d q_{n-1} \wedge d p_{n} \neq 0 .
$$

Now the corollary follows from Theorem 5 , where we put $f \equiv 1$.

REMARK 3. Now we suppose that $\operatorname{rank} \omega_{x}<2(n-1)$. Then there are two possibilities provided the assumptions of Theorem 5 are satisfied.

(A) There exists a germ of a 1-form $\theta$ at $x$ such that $\omega=d \theta$ and $\theta$ has a constant Pf-rank $r(r<n-1)$. Then we can take $f \equiv 1$ and $\alpha=\theta$.

(B) The 1-form $\theta$ defined in (A) does not exist, but there exists a smooth functiongerm $f$ and a germ of a smooth 1-form $\alpha$ such that $\omega=d(f \alpha)$ and $\alpha$ has a constant Pf-rank $r>0$.

Now we explain the case (B). We have $\omega=d f \wedge \alpha+f d \alpha$,

$$
\omega^{r} \wedge(f \alpha)=\left[r f^{r-1} d f \wedge \alpha \wedge(d \alpha)^{r-1}+f^{r}(d \alpha)^{r}\right] \wedge(f \alpha)=f^{r+1}(d \alpha)^{r} \wedge \alpha
$$

and

$$
\omega^{r+1} \wedge(f \alpha)=f^{r+2}(d \alpha)^{r+1} \wedge \alpha \equiv 0 .
$$

If $\left.\omega\right|_{x} ^{r} \wedge\left(\left.f(x) \alpha\right|_{x}\right) \neq 0$ then $\omega^{r} \wedge(f \alpha) \neq 0$ on some neighborhood $V$ of $x$ which means that $\theta:=f \alpha$ has a constant Pf-rank $r$. This contradicts the assumption of (B). Consequently $\left.\omega\right|_{x} ^{r} \wedge\left[\left.f(x) \alpha\right|_{x}\right]=0$. Since $\left.\left.(d \alpha)\right|_{x} ^{r} \wedge \alpha\right|_{x} \neq 0$, we see that $f$ has to vanish at $x$, and therefore $\left.\theta\right|_{x}=\left.f(x) \alpha\right|_{x}=0$. In this case we should investigate the set $\mathcal{M}_{x}^{\omega}$ of germs in $x$ of all smooth 1-forms $\theta$ such that $d \theta=\omega$ and $\left.\theta\right|_{x}=0$.

4. Maximal isotropic germs in the singular symplectic structures. Let $\omega$ be germ at zero of a closed 2-form on $R^{2 n}$. Let $(L, 0) \subset R^{2 n}$ be germ of a smooth submanifold of $R^{2 n}, \operatorname{dim} L=n$, such that $\left.\omega\right|_{L}=0$. If $\omega$ is a symplectic form, then $(L, 0)$ is called the Lagrangian germ. If $\omega$ is a singular symplectic form, then $(L, 0)$ will be called an Misotropic germ (maximal isotropic germ). One of the fundamental problems of symplectic geometry (cf. [17]) is to study the local and global structure of the space of Lagrangian submanifolds. There is a fundamental application of the singular symplectic structures in 
physics of equilibrium processes (cf. [11]) and electromagnetic interactions (cf. [5]). Again there is a very natural motivation for investigation of isotropic subspaces and especially maximal isotropic subspaces, which correspond to equilibrium states of the degenerate systems.

The natural classification groups for isotropic submanifold-germs are those which preserve $\omega$. There is a crucial difference between the singular symplectic case and the symplectic case, where all germs of Lagrangian submanifolds are equivalent. If the singularity set of $\omega$ is not empty then even the situation, when there exist no M-isotropic germ, may happen. Now we present an example of such situation.

ExAmPLE 2. Let $c: R \rightarrow R^{4}$ be a curve given by the formula

$$
c(t)=\left(c_{1}(t), c_{2}(t), c_{3}(t), c_{4}(t)\right) \text {, }
$$

where

$$
\begin{array}{rlrl}
c_{1}(t) & :=\frac{1}{2} \cos t(1+\cos t), & c_{2}(t) & :=\frac{1}{2} \sin t(1+\sin t), \\
c_{3}(t) & :=-\frac{1}{2} \cos t(1-\cos t), \quad c_{4}(t):=-\frac{1}{2} \sin t(1-\sin t), \quad t \in R .
\end{array}
$$

We define a 1 -form $\alpha$ on $R^{4}$ by the formula

$$
\begin{gathered}
\alpha(x):=e^{-1 / r^{2}}\left(\sum_{k=1}^{4} x_{k} c_{k}\left(\frac{1}{r}\right)\right) d r, \quad x \in R^{4} \backslash\{0\}, \\
\alpha(0):=0
\end{gathered}
$$

where $x=\left(x_{1}, x_{2}, x_{3}, x_{4}\right)$ and $r^{2}(x):=\|x\|^{2}=\sum_{k=1}^{4} x_{k}^{2}$. It is clear that $\alpha$ is smooth (is a $C^{\infty}$-form) on $R^{4}$. Let $\omega:=d \alpha$. Then $\omega$ is a smooth closed 2 -form on $R^{4}$ and

$$
\begin{aligned}
\omega=\sum_{k=1}^{4} x_{k} \frac{d}{d r}\left[e^{-1 / r^{2}} c_{k}\left(\frac{1}{r}\right)\right] d r \wedge d r+\sum_{k=1}^{4} e^{-1 / r^{2}} c_{k}\left(\frac{1}{r}\right) d x_{k} \wedge d r & \\
& =e^{-1 / r^{2}}\left(\sum_{k=1}^{4} c_{k}\left(\frac{1}{r}\right) d x_{k}\right) \wedge d r .
\end{aligned}
$$

Let $S$ be an arbitrary smooth surface (a 2-dimensional submanifold) in $R^{4}$ such that $0 \in S$. Let $\gamma:(-\epsilon ; \epsilon) \rightarrow S$ be an arbitrary geodesic line (with respect to the Riemann structure defined on $S$ by the standard scalar product in $R^{4}$ ) parametrized by the natural parameter (the arc length) and such that $\gamma(0)=0$. Denote by $\dot{\gamma}$ the velocity vector field along $\gamma\left(\dot{\gamma}(t)=\frac{d \gamma}{d t}(t)\right)$ and by $v$ the normalized vector field along $\gamma$ tangent to $S$ and perpendicular to $\frac{\partial}{\partial r} \circ \gamma$ for $t \neq 0$. (For $\epsilon$ sufficiently small there exist exactly two such vector fields. Then we choose one of them.) We have $d r(v(t))=0$ and therefore

$$
\begin{aligned}
& \omega(v(t), \dot{\gamma}(t))=e^{-1 / r^{2}}\left(\sum_{k=1}^{4} c_{k}\left(\frac{1}{r}\right) d x_{k}(v) d r(\dot{\gamma}(t))-\sum_{k=1}^{4} c_{k}\left(\frac{1}{r}\right) d x_{k}(\dot{\gamma}(t)) d r(v)\right) \\
& =e^{-1 / r^{2}(t)} \sum_{k=1}^{4} c_{k}\left(\frac{1}{r(t)}\right) v_{k}(t)[\dot{\gamma}(t)](r)=e^{-1 / r^{2}(t)} \sum_{k=1}^{4} c_{k}\left(\frac{1}{r(t)}\right) v_{k}(t)\left\langle\dot{\gamma}(t), \frac{\gamma(t)}{|\gamma(t)|}\right\rangle
\end{aligned}
$$


for $t \neq 0$, where $r(t):=|\gamma(t)|, v_{k}(t)=d x_{k}(v(t))$ is the $k$-th coordinate of the vector $v(t)$ and $\langle\cdot, \cdot\rangle$ denotes the standard scalar product in $R^{4}$. Since $\lim _{t \rightarrow 0^{+}} \frac{\gamma(t)}{|\gamma(t)|}=\dot{\gamma}(0)$ we obtain

$$
\lim _{t \rightarrow 0^{+}}\left\langle\dot{\gamma}(t), \frac{\gamma(t)}{|\gamma(t)|}\right\rangle=|\dot{\gamma}(0)|^{2}=1 \text {. }
$$

Then there exists $\delta_{1}>0$ such that for any $0<t<\delta_{1}$ we have $\left\langle\dot{\gamma}(t), \frac{\gamma(t)}{|\gamma(t)|}\right\rangle>0$. Since $\lim _{t \rightarrow 0^{+}} v(t)=v(0)$ and $|v(0)|=1$ then there exist $\delta_{2}>0$ and a natural number $0 \leq k \leq 4$ such that for any $0<t<\delta_{1}$ we have $v_{k}(t) \neq 0$. Hence there exist $n \in \mathbf{N}$ and $0<t_{n}<\delta:=\max \left\{\delta_{1}, \delta_{2}\right\}$ such that

$$
r\left(t_{n}\right)=\left|\gamma\left(t_{n}\right)\right|=\frac{1}{(2 n+k / 2) \pi}<\delta .
$$

Consequently

$$
\omega\left(v\left(t_{n}\right), \dot{\gamma}\left(t_{n}\right)\right)=e^{-1 / r^{2}\left(t_{n}\right)} v_{k}\left(t_{n}\right)\left\langle\dot{\gamma}\left(t_{n}\right), \frac{\gamma\left(t_{n}\right)}{\left|\gamma\left(t_{n}\right)\right|}\right\rangle \neq 0
$$

which means that $S$ is not an isotropic (Lagrange) submanifold of $\omega$ in $R^{4}$. Hence there are no Lagrange submanifolds of $\omega$ containing the origin of $R^{4}$. This also implies that $\omega$ is not of the form (15).

On the other hand, if we put

$$
\Phi(x):= \begin{cases}\left(e^{-1 / r}\left(\sum_{k=1}^{4} x_{k} c_{k}\left(\frac{1}{r}\right)\right), 0, f(r), 0\right) & \text { for } x \neq 0, \\ (0,0,0,0) & \text { for } x=0,\end{cases}
$$

where $r=|x|$ and $f(r):=\int_{0}^{r} e^{-1 / t} d t$, then $\Phi$ is smooth and $\omega=\Phi^{*} \omega_{D}$.

Now we show that even in the very degenerate case there is a possibility for a classification of the generic maximal isotropic germs.

EXAmple 3. Let $\omega$ be a germ of a closed 2-form on $R^{4}$ at $0 \in R^{4}$. Then $\omega^{2}=f \Omega$, where $\Omega$ is a germ of a volume form on $R^{4}$ at 0 and $f$ is a function-germ at 0 . We assume that $f(0)=0,\left.d f\right|_{0} \neq 0$ and $\iota^{\star} \omega=0$, where

$$
\iota: \Sigma_{2} \rightarrow R^{4} \text {. }
$$

By Corollary 2, there exists a germ of a diffeomorphism $\Phi:\left(R^{4}, 0\right) \rightarrow\left(R^{4}, 0\right)$ such that

$$
\Phi^{\star} \omega=d\left(p_{1}\left(d q_{1}+q_{2} d p_{2}\right)\right) .
$$

Let $L$ be a germ of maximal isotropic submanifold in $\left(R^{4}, \omega\right)$. If $L$ is transversal to $\Sigma_{2}=\left\{p_{1}=0\right\}$ at 0 then it is easy to see that

$$
L=\left\{(p, q) \in R^{4}: q_{1}=f_{1}(p), q_{2}=f_{2}(p)\right\}
$$

(up to diffeomorphisms which preserve $\omega$ ), where $f_{1}, f_{2}$ are function-germs on $R^{2}$ at 0 , which vanish at 0 . $L$ is isotropic, therefore $d\left(p_{1}\left(d f_{1}+f_{2} d p_{2}\right)\right)=0$. By the same method as in the proof of Lemma 2 it is easy to prove that $p_{1}\left(d f_{1}+f_{2} d p_{2}\right)=d\left(p_{1}^{2} F\right)$, where $F$ is a function-germ on $R^{2}$ at 0 . Hence

$$
L=\left\{(p, q) \in R^{4}: q_{1}=-S(p)-p_{1} \frac{\partial S}{\partial p_{1}}(p), q_{2}=\frac{\partial S}{\partial p_{2}}(p)\right\},
$$


where $S$ is a function-germ on $R^{2}$ at 0 , vanishing at 0 and $F=-\frac{\partial S}{\partial p_{1}}$. We call $S$ a generating function-germ for $L$.

By a diffeomorphism of the form $\left(p_{1}, p_{2}, q_{1}, q_{2}\right) \mapsto\left(p_{1}, p_{2}, q_{1}-f_{1}(p), q_{2}-f_{2}(p)\right)$, which preserves $\omega$, one can reduce $L$ to the following germ of maximal isotropic submanifold

$$
L_{0}=\left\{(p, q) \in R^{4}: q_{1}=0, q_{2}=0\right\} .
$$

Obviously the germs of maximal isotropic submanifolds that are not transversal to $\Sigma_{2}$ at 0 are not equivalent to $L_{0}$.

Let $\omega$ be the pullback of the Darboux form $\omega_{D}$

$$
\omega=\Phi^{\star} \omega_{D},
$$

where $\Phi:\left(R^{2 n}, 0\right) \rightarrow\left(R^{2 n}, 0\right)$ is a smooth irregular map-germ. We consider the group $\mathcal{G}_{\omega}$ of diffeomorphism-germs preserving $\omega$, i.e. $h^{*} \omega=\omega$ if $h \in \mathcal{G}_{\omega}$. By $\mathcal{S}_{\omega}$ we denote those elements of $\mathcal{G}_{\omega}$ which are $\Phi$-lowerable to a symplectomorphism, i.e. $h \in \mathcal{S}_{\omega}$ if there exists a symplectomorphism-germ $\phi:\left(R^{2 n}, \omega_{D}\right) \rightarrow\left(R^{2 n}, \omega_{D}\right)$ such that

$$
\Phi \circ h=\phi \circ \Phi \text {. }
$$

The aim of this section is to provide the classification of M-isotropic germs under the $\mathcal{S}_{\omega}$-group action. The classification of M-isotropic germs for the subgroup of $\mathcal{S}_{\omega}$ of diffeomorphism germs preserving an extra canonical fibration was presented in [12] for the $\Sigma_{20}$ Martinet's singular form $\omega$. Here we extend this result for the whole $\mathcal{S}_{\omega}$-group action in the case of the $\Sigma_{20}$ Martinet's singular symplectic form

$$
\omega=p_{1} d p_{1} \wedge d q_{1}+\sum_{i=2}^{n} d p_{i} \wedge d q_{i} .
$$

It is easy to see that $\omega=\Phi^{*} \omega_{D}$, where $\Phi(p, q)=\left(\frac{1}{2} p_{1}^{2}, p_{2}, \ldots, p_{n}, q_{1}, \ldots, q_{n}\right)$.

Definition 2. The M-isotropic germ $N \subset\left(R^{2 n}, \omega\right)$ is called $\Phi$-lowerable if there exists a Lagrangian germ $L \subset\left(R^{2 n}, \omega_{D}\right)$ such that $\Phi^{-1}(L) \subset N$.

Proposition 4. All smooth $M$-isotropic germs in $\left(R^{2 n}, \omega\right)$ are $\Phi$-lowerable.

Proof. The proposition follows immediately from the proof of Proposition 2.7 in [12]. In fact any smooth M-isotropic germ $(N, 0)$ may be written in the form

$$
q_{i}=\left.\frac{\partial F}{\partial \xi_{i}}(\lambda, \xi)\right|_{\xi=\left(\frac{1}{2} p_{1}^{2}, \ldots, p_{n}\right)}, \quad 0=\left.\frac{\partial F}{\partial \lambda_{j}}(\lambda, \xi)\right|_{\xi=\left(\frac{1}{2} p_{1}^{2}, \ldots, p_{n}\right)},
$$

where $1 \leq i \leq n, 1 \leq j \leq k,(\lambda, \xi) \rightarrow F(\lambda, \xi)$ is a generating Morse family-germ for a Lagrangian germ $(L, 0)$ of $\left(R^{2 n}, \omega_{D}\right)$. There is an $\mathcal{S}_{\omega}$-equivalent representation of M-isotropic germ by the Legendre transformation of the function $F$ (cf. [17], [3]), namely

$$
\frac{1}{2} p_{1}^{2}=\frac{\partial G}{\partial q_{1}}(\lambda, q), \ldots, p_{n}=\frac{\partial G}{\partial q_{n}}(\lambda, q), \quad 0=\frac{\partial G}{\partial \lambda}(\lambda, q) .
$$

This form is accessible for the M-isotropic germs $(N, 0) \subset\left\{p_{1}=0\right\}$.

By the action of the $\mathcal{S}_{\omega}$-group of equivalences of M-isotropic germs we get the following classification result. 
TheOREM 6. Any smooth $M$-isotropic germ of $\left(R^{2 n}, \omega\right)$ is $\mathcal{S}_{\omega}$-equivalent to

$$
(N, 0)=\left(\left\{(p, q): q_{i}=0,1 \leq i \leq n\right\}, 0\right)
$$

or

$$
(N, 0)=\left(\left\{(p, q): p_{i}=0,1 \leq i \leq n\right\}, 0\right) .
$$

Proof. All smooth M-isotropic germs are represented by the Lagrangian germs. These Lagrangian germs are transversal to the hypersurface $\left\{p_{1}=0\right\}$ or they are contained in this hypersurface. If the germ $(L, 0)$ is generated by $F$ from the equations $(19)$ then it may be reduced to $F \equiv 0$ by a $\Phi$-liftable symplectomorphism of $\left(R^{2 n}, \omega_{D}\right)$ (cf. [6], Theorem 1 ). If the germ $(L, 0)$ is represented by a function $G$ (it includes the case when $(L, 0)$ is contained in $\left.\left\{p_{1}=0\right\}\right)$ then it may be reduced to $G \equiv 0$. So the corresponding lifted elements of $\mathcal{S}_{\omega}$ reduce the M-isotropic germ to $N=\{(p, q): q=0\}$ or to $N=$ $\{(p, q): p=0\}$.

The canonical singular M-isotropic germs, i.e. the singular M-isotropic map-germs which are not enclosed into $\left\{p_{1}=0\right\}, \iota: R^{n} \rightarrow\left(R^{2 n}, \omega\right), \iota^{*} \omega=0$, are represented by the Lagrangian germs $(L, 0)$, which are not transversal to the hypersurface $\left\{p_{1}=0\right\}$. By the straightforward use of Theorem 3 in [6] and further reduction of the prenormal forms introduced in [18] (Theorem 1), the corank 1 singular M-isotropic germs are classified in the following theorem.

TheOREM 7. Let $\omega$ be a germ of the $\Sigma_{20}$ Martinet's singular form.

A. The prenormal forms of the generic $M$-isotropic germs of $\left(R^{2 n}, \omega\right)$ represented by nontransversal Lagrangian germs are given in the form

$$
\frac{1}{2} p_{1}^{2}=\frac{\partial S}{\partial q_{1}}(q), p_{2}=\frac{\partial S}{\partial q_{2}}(q), \ldots, p_{n}=\frac{\partial S}{\partial q_{n}}(q),
$$

where

$$
\tilde{A}_{k}: \quad S(q)= \pm q_{1}^{k+1}+\sum_{i=2}^{k} g_{i}\left(q_{2}, \ldots, q_{n}\right) q_{1}^{k+1-i},
$$

and $g_{k} \in \mathbf{m}^{2}$ ( $\mathbf{m}$ is a maximal ideal of the ring of smooth function-germs at zero).

B. The simple normal forms of $M$-isotropic germs of $\left(R^{2 n}, \omega\right)$ are $\mathcal{S}_{\omega}$-equivalent to one represented by function $y \rightarrow S(q)$ in the form

$$
\begin{aligned}
& A_{k}: S(q)=q_{1}^{3}+q_{1}\left( \pm q_{2}^{k+1}+Q\left(q_{3}, \ldots, q_{n}\right)\right), \\
& D_{k}: S(q)=q_{1}^{3}+q_{1}\left(q_{2}^{2} q_{3} \pm q_{3}^{k-1}+Q\left(q_{4}, \ldots, q_{n}\right)\right), k \geq 4, \\
& E_{6}: S(q)=q_{1}^{3}+q_{1}\left(q_{2}^{3} \pm q_{3}^{4}+Q\left(q_{4}, \ldots, q_{n}\right)\right), \\
& E_{7}: S(q)=q_{1}^{3}+q_{1}\left(q_{2}^{3}+q_{2} q_{3}^{3}+Q\left(q_{4}, \ldots, q_{n}\right)\right), \\
& E_{8}: S(q)=q_{1}^{3}+q_{1}\left(q_{2}^{3}+q_{3}^{5}+Q\left(q_{4}, \ldots, q_{n}\right)\right), \\
& C_{k}: S(q)=q_{1}^{2 k+1}+q_{1}^{2} q_{2}+q_{1} Q\left(q_{3}, \ldots, q_{n}\right), k \geq 2, \\
& B_{k}: S(q)=q_{1}^{3} q_{3}+q_{1}^{2} q_{2}+q_{1}\left( \pm q_{3}^{k}+Q\left(q_{4}, \ldots, q_{n}\right)\right), k \geq 2, \\
& F_{4}: S(q)=q_{1}^{5}+q_{1}^{2} q_{2}+q_{1}\left(q_{3}^{3}+Q\left(q_{4}, \ldots, q_{n}\right)\right), \\
& U_{3}: S(q)= \pm q_{1}^{4}+q_{1} Q\left(q_{2}, \ldots, q_{n}\right) .
\end{aligned}
$$




\section{References}

[1] V. I. Arnol'd, Critical points of functions on a manifold with boundary, the simple Lie groups $B_{k}, C_{k}$ and $F_{4}$, and singularities of evolutes, Uspekhi Mat. Nauk 33:5 (1978), 91-105 (Russian); English transl.: Russian Math. Surveys 33:5 (1978), 99-116.

[2] V. I. Arnol'd, A. B. Givental', Symplectic Geometry, Current Problems in Mathematics, Fundamental Directions 4, Itogi Nauki i Tekhniki, VINITI, Moskva, 1985, 5-139 (Russian); English transl.: Dynamical systems IV, 1-138, Encyclopaedia Math. Sci. 4, Springer, Berlin, 2001.

[3] V. I. Arnol'd, S. M. Guseǔn-Zade, A. N. Varchenko, Singularities of Differentiable Maps I, Monogr. Math. 82, Birkhäuser, Boston, 1985.

[4] R. L. Bryant, S. S. Chern, R. B. Gardner, H. L. Goldschmidt, P. A. Griffiths, Exterior Differential Systems, Math. Sci. Res. Inst. Publ. 18, Springer, New York, 1991.

[5] Y. Choquet-Bruhat, Géométrie différentielle et systèmes extérieurs, Dunod, Paris, 1968.

[6] W. Domitrz, S. Janeczko, Equivalence of Lagrangian germs in the presence of a surface, in: Symplectic Singularities and Geometry of Gauge Fields, Banach Center Publ. 39, Warsaw, 1997, 31-37.

[7] W. Domitrz, S. Janeczko, Normal forms of symplectic structures on the stratified spaces, Colloq. Math. 68 (1995), 101-119.

[8] M. Golubitsky, D. Tischler, An example of moduli for singular symplectic forms, Invent. Math. 38 (1977), 219-225.

[9] B. Jakubczyk, M. Zhitomirskii, Local reduction theorems and invariants for singular contact structures, Ann. Inst. Fourier (Grenoble) 51 (2001), 237-295.

[10] B. Jakubczyk, M. Zhitomirskii, Odd-dimensional Pfaffian equations: reduction to the hypersurface of singular points, C. R. Acad. Sci. Paris Sér. I Math. 325 (1997), 423-428.

[11] S. Janeczko, Singularities of Lagrangian varieties and related topics, Acta Appl. Math. 25 (1991), 21-57.

[12] S. Janeczko, A. Kowalczyk, On singularities in the degenerated symplectic geometry, Hokkaido Math. J. 19 (1990), 103-123.

[13] J. Martinet, Sur les singularités des formes différentielles, Ann. Inst. Fourier (Grenoble) 20 (1970), 95-178.

[14] J. Martinet, Singularities of Smooth Functions and Maps, London Math. Soc. Lecture Note Ser. 58, Cambridge Univ. Press, Cambridge, 1982.

[15] J. Moser, On the volume elements on a manifold, Trans. Amer. Math. Soc. 120 (1965), 286-294.

[16] R. Roussarie, Modèles locaux de champs et de formes, Astérisque 30 (1975), 1-181.

[17] A. Weinstein, Lectures on Symplectic Manifolds, CBMS Regional Conf. Ser. in Math. 29, Amer. Math. Soc., Providence, 1977.

[18] V. M. Zakalyukin, O. M. Myasnichenko, Lagrangian singularities under symplectic reduction, Funktsional. Anal. i Prilozhen. 32:1 (1998), 1-11; English transl.: Funct. Anal. Appl. 32 (1998), 1-9. 